\title{
OPTIMAL NARROWBAND AND BROADBAND DATA COMMUNICATION TRANSMISSION IN LOW-VOLTAGE POWER LINE
}

\author{
Ndolo A.O. ${ }^{1}$ \\ ${ }^{1}$ Tutorial \& Research Fellow, Electrical and Electronic Engineering Department, Machakos University College \\ (A Constituent College of Kenyatta University), Republic of Kenya \\ tonindolo@machakosuniversity.ac.ke
}

\begin{abstract}
We investigate the usage of low-voltage electrical power supply network as a communication channel for the provision of data communication services. This is done through simulations and measurements of the power line channel transfer function in the frequency band of 0-30 MHz. A Parallel Resonant Circuit $(P R C)$ channel model is then proposed to model the power line network and results compared to a Series Resonant Circuit (SRC) model. The model is simulated and validation done through measurements on a test bed for a cascaded network with three T-node branches. Transverse Electromagnetic (TEM) transmission line resonant circuit theory is employed to calculate and optimize the obtained parameters of the model. From the results obtained, the simulated models and measurement results are confirmed to compare very well with each other, and thus the proposed PRC model is considered appropriate for the characterization and modelling of the power line network channel.
\end{abstract}

Keywords: Transmission Line (TL), Power line network (PLN), Power line communication (PLC), Channel Modelling \& Simulation, Function Transfer, Resonant Circuits, TEM mode, Parallel Resonant Circuit (PRC), Series Resonant Circuit (SRC)

\section{INTRODUCTION}

The electrical power supply networks are viewed as alternative channels for broadband data transmission due to increased demand for data communication and information. This is attributed to the fact that the electrical power supply network infra-structure is in existence and less cost is incurred in the set up and installation. As a result, the electrical power line is transformed into a multifunctional medium for the delivery of electrical energy, voice, and video and data information. Because of the considerable difference in topology, structure and physical properties from the normal and conventional wired transmission mediums like fibre optical cables [1],[17], the electrical power line network presents a very harsh and unfavorable environment for high frequency digital communication. In the recent research and development of the low-voltage electrical power line network for communication of high frequency data signals, various modelling techniques have been used to characterize such network with design parameters being informed by the properties of the channel transfer characteristics and capacity offered by the channel [1],[2],[3],[17]. This calls for accurate models that can best describe the characteristics and transmission behavior over the PLC with adequate precision [2],[4],[17],[18]. Currently, there is no universal standard model for the PLC. The two common approaches used to model the power line network are the top-down and bottom-up methods. The models presented in [3],[6], among other researchers, employs the top-down approach by using measurement data to model and calculate the parameters of the models. In [1], [2], [4], [7],[8],[18] the bot- tom-up approach is used where the PLC is characterized, parameters calculated and models defined.

The paper's objective is to investigate and model the power line characteristics as a channel for data communication in the frequency band of $0-30 \mathrm{MHz}$ using the bottom-up method. Transmission line parallel resonant circuit is used to obtain the model parameters. In [2], a series resonant circuit model is presented and transmission line theory is used to calculate the parameters of the model, while in [5],[6] an evolutionary approach is employed to obtain the optimized component parameters of the presented series resonant circuit model. In this research, we use the power line cable primary parameters that were obtained and presented in [10] with an assumption that the cable supports only TEM mode signal propagation only. These parameters include: power line cable characteristic impedance, $\mathrm{Z}$, propagation constant, $\gamma$, attenuation constant, $\alpha$, and propagation velocity $v$. This paper has analysis of transmission line parallel resonant circuit is presented and the effects of short circuited transmission lines studied. The obtained parallel resonant circuit function transfer is analyzed and presented. Simulation and measurement results are presented to evaluate the performance of the model. Analysis and discussion of the results is discussed. 


\section{TRANSMISSION LINE RESONANT CIRCUIT}

At high frequencies of signal transmission, the usual lumped LC resonant circuits are replaced by open or short circuited sections of transmission lines [11], [12], [13], [18]. By analyzing the input impedance characteristics around the resonant wavelengths of the circuits of Figure 1, we can confirm that these circuits behave and possess the characteristics of a parallel resonant circuit.

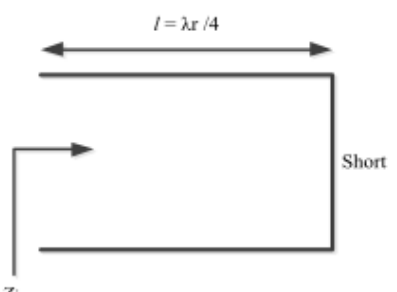

(a)

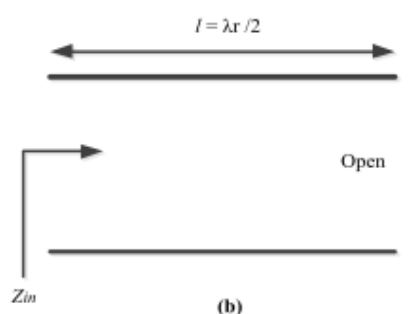

(b)
Figure 1. Types of transmission line parallel resonant circuits with length (a) a quarter wavelength. (b) a half wavelength [12], [13], [18]

In this section, the study and quantitative analysis of a resonant transmission line section is presented. We derive the parameters of the model with an assumption that the cable supports TEM mode signal propagation only [10], [14], [18].

\subsection{Parallel Resonant Circuit Model}

A practical transmission line cable is as shown in Figure 1. This line will behave as a parallel resonant circuit when the length is in odd multiples of $\lambda_{r} / 4$ or in even multiples of $\lambda_{r} / 2$. The input impedance $Z_{\text {in }}$ from Figure 1 that will be seen by the signal is given as [11],[12],[13],[18]:

$Z_{\text {in }}=Z_{0} \frac{\alpha l+j \frac{\pi \delta \omega}{\omega_{0}}}{1+j \alpha l \frac{\pi \delta \omega}{\omega_{0}}}$

The assumption in (1) is that $\alpha l \frac{\pi \delta \omega}{\omega_{0}} \ll 1$

Therefore, a $\lambda / 4$ long transmission line at the resonant frequency, will be [11], [12][13],[18]:

$$
Z_{\text {in }} \approx Z_{0}
$$

Now, using an equivalent parallel resonant circuit shown in Figure 2 below, the frequency dependent input admittance, $Y_{\text {in }}(f)$ seen by the input signal is given in [11], [12], [13], [18]:

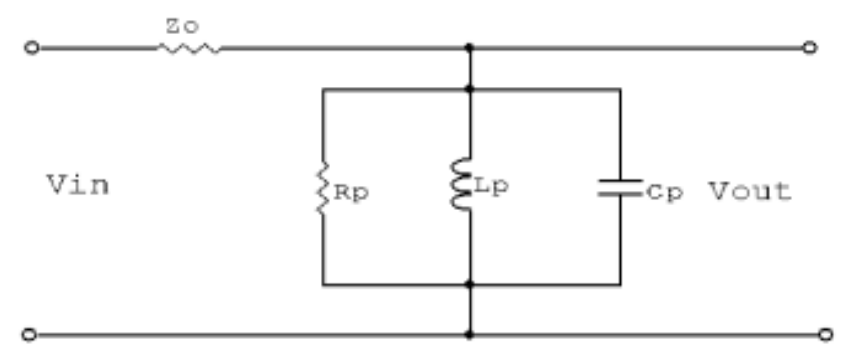

Figure 2. Equivalent Parallel Resonant Circuit [14],[17],[18].

$$
Y_{i n}=\frac{1}{R_{p}}+j \omega C_{p}+\frac{1}{j \omega L_{p}}
$$

where $Z_{p}, R_{p}, L_{p}$ and $C_{p}$ are the parallel input impedance, resistance, inductance and capacitance respectively. For a loss prone circuit, the input impedance will be equal to $1 / R_{p}$ at resonance. For a $\lambda / 2$ open circuited transmission line, the input impedance can be approximated in [11],[12],[13], [18]:

$$
z_{\text {in }}=\frac{z_{0}}{\alpha l+j \frac{\pi \delta \omega}{\omega_{0}}}
$$

From these equations the following can be computed, $\mathrm{Z}_{0}$ characteristic impedance of the power line cable, $\lambda_{\mathrm{r}}$ resonance wavelength, $\alpha$ - attenuation constant, $R_{p}$ - parallel resistance, $L_{p}$ - parallel inductance, $C_{p}$ - parallel capacitance, $\mathrm{Q}$ - resonance quality factor, $\beta_{\mathrm{r}}=\pi / \mathrm{L}$ - phase constant at resonance and $\omega_{0}$ - angular resonance frequency.

\section{MODELLING OF CHANNEL IN REQUENCY DOMAIN}

\subsection{Model Cable Lengths and Notch Positions}

To establish the position and separation of the notches or resonant points along the transfer functions, we consider the electric length of the branches. A relationship expressed in terms of the wavelength, $\lambda$, propagation velocity, $v_{p}$ and frequency, $\mathrm{f}$.

For open circuit ended branches, the first notch will occur at a resonant frequency relative to the branch length, $\mathrm{L}$ given in [2], [16],[18]:

$$
f_{0}=\frac{v_{p}}{f}
$$

The following notches or resonant points along the open circuit branches are given by (6), while for the short circuit ended branches, the first notch occurs at zero and subsequent frequencies are given by (7) in [2], [16]. 
$f_{0 k}=\frac{v_{p}(2 k+1)}{4 l}$

$$
f_{s k}=\frac{v_{p}(2 k)}{4 l}
$$

Where $\mathrm{k}=1,2,3 \ldots \ldots, v_{p}=1.49 \times 10^{8} \mathrm{~m} / \mathrm{s}$ and $\mathrm{L}$ is the length of the branch.

For a cascaded network configuration, the function transfer will be observed to follow a trend of addition in the function transfer. Thus the function transfer can be calculated illustrated in [17], [18].

$$
H_{\text {total }}=H_{\text {conf } 1}+H_{\text {conf } 2}+H_{\text {conf } 3}+\ldots \ldots \ldots . .+H_{\text {conf }}[d B]
$$

$\mathrm{N}=1,2,3, \ldots \ldots \ldots$

\subsection{PRC Transfer Function}

Having obtained the frequency dependent input impe-dance, $Z_{p}(f)$ in (3), the transfer function $h_{r i}(f)$ for each resonant circuit or point along the frequency span for a given length [17], [18]:

$$
h_{r i}(f)=\frac{V_{\text {out }}}{V_{\text {in }}}=\frac{Z_{0}}{Z_{0}+Z_{p}(f)}
$$

Thus for $\mathrm{n}$ resonant circuits in a branch length, the transfer function $H_{r i}(f)$ can be determined [17], [18]:

$$
H_{r i}(f)=\prod_{i=1}^{n} h_{r i}(f)
$$

Therefore, the overall network transfer function $H(f)$ is calculated by the product of all the branch transfer function which can be expressed as:

$$
H(f)=B \prod_{i=1}^{N} H_{r i}(f)
$$

Hence, $\mathrm{N}$ is the number of branches in the network and $\mathrm{B}$ is the direct path attenuation factor from the transmitter to the receiver [2], [3]:

$$
B(f, l)=e^{-(\alpha+j \beta) l}=e^{-\alpha l}
$$

where $\alpha$ and $\beta$ are the attenuation and phase constants of the power line from the propagation constant, $f$ is the frequency of the signal and $l$ is the direct path length from the transmitter to the receiver.

\section{SIMULATION AND MEASUREMENT}

Figure 3 is schematic wiring diagram of the test bed network used, while Figure 4 shows results.

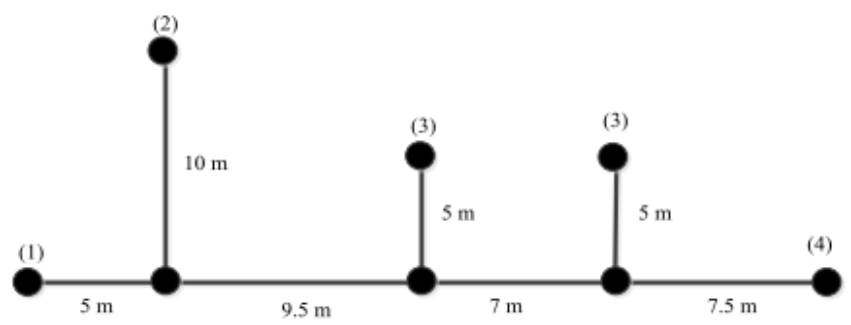

Figure 3. Cascade three T nodes branch network

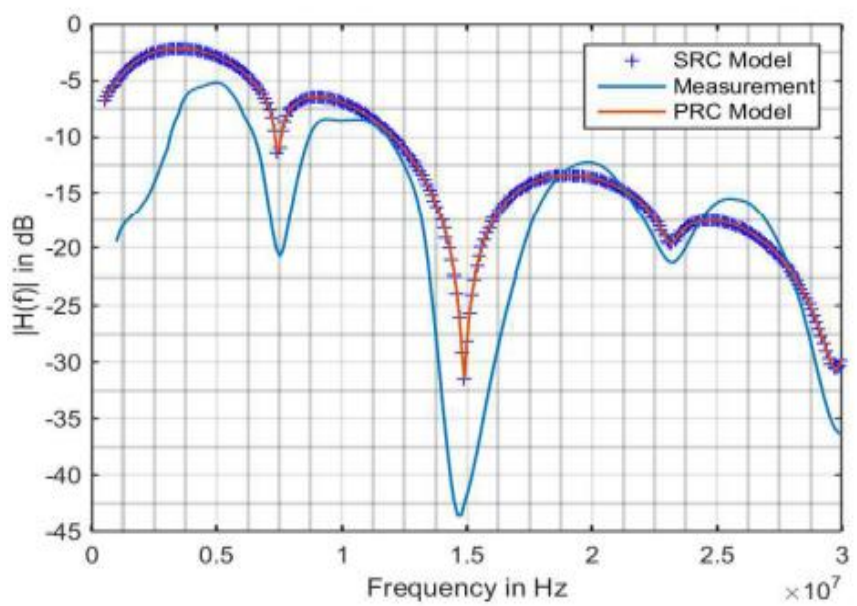

Figure 4. Configuration measurement and results for the network Figure 3.

\section{RESULTS' SUMMARY AND CONCLUSION}

From the transfer function results, it is observed that the number and depth of notches along the transfer function is dependent on the length of the branch. That is, the longer the branch, the more the number of notches with low depths as compared to shorter branch lengths that have few number of notches but with more pronounced deep notches. It can also be observed that, having successive branches of the same length results in more pronounced and wider notches. This is not a desirable effect for data transmission as the signal will be subjected to severe loss. Thus the operation band should be chosen such that notches are avoided. The transfer function is seen as a cascade of the amplitude responses for individual resonant circuits which correlates well with both the simulations and measurement results presented here. This is because of the multiple cascaded single resonant points along the cable as the signal propagates. In this research, we employ the transmission line equivalent PRC to obtain the optimized values for the RLC component parameters. It is worth noting that as the frequency and branch length increases, the signal amplitudes are consequently attenuated. This implies that attenuation in the power line channel is a factor of frequency and cable length. In our research, the PLC channel has been characterized and studied as a transmission line composed of a cascade of parallel resonant circuits connected to a line of known 
characteristic impedance. Based on this characterization, a PRC model is presented. A network is built based on the simulated model and measurements done for validation. Simulation and measurement results compare very well with the SRC model results and therefore the power line network has been successfully modelled as a communication channel using the PRC model. The PLC channel transfer function is observed to have notches whose position, depth and width are dependent on the branch length, load and characteristics of the power line.

\section{REFERENCES}

[1]. Kruk, B.I., Nopantonopylo V.N., Shuvalov V.N., "Telecommunication Systems and Networks, Modern Technologies", Garyachi-Liniya Telekom publishers, Moscow, Russian Federation, 2003 (Russian script).

[2]. Krukhmalyev, V.V., Gor'diyentko V.N., Mochenov A.D., " Digital Transmission Systems", Garyachi-Liniya Telekom publishers, Moscow, Russian Federation, 2007 (Russian script).

[3]. Alekseev L.B. Znamenskyi A.E., Polyakov V.S. "Microwave filters and Circuits" Syvazi Publishers, Moscow, Russian Federation, 1976. (Russian script)

[4]. N. L. Zaw, H. A. Kyaw, and K. Z. Ye, "Power line cable transfer function for the broadband power line communication channel," Universal Journal of Control and Automation, vol. 1, no. 4, pp. 103-110, 2013.

[5]. Ledovckyi A., Ruduinskyi N.I. "Radar and Radio Communication System", Garyachi-Liniya Telekom publishers, Moscow, Russian Federation, 2006 (Russian script).

[6]. H. Philips, "Modelling of power line communication channels," in Proceedings of Int. Symposium on Power-Line Communications and Its Application, ser. Vancouver, Canada, 6-8, Apr. 2005, pp. 14-21.

[7]. S. Galli, "A simplified model for the indoor power line channel," in Proceedings of IEEE Int. Symposium on Power-Line Communications and Its Applications, ser. Dresden, Germany, 29 Mar.-1 Apr. 2009, pp. 13-19.

[8]. P. Mlynek, J. Misurec, M. Koutny, and R. Fujdiak, "Transfer function of power line channel-influence of topology parameters and power line topology estimation," in Proceedings of IEEE PES Innovative Smart Grid Technologies Conference, ser. Istanbul, 12-15, Oct. 2014, pp. 1-5.

[9]. R. E. Collin, Foundations for Microwave Engineering, 2nd ed. John Wiley \& Son, Inc., 2001.

[10]. D. M. Pozar, Microwave Engineering, 2nd ed. John Wiley \& Sons Inc., 1998.

[11]. D. K. Misra, Radio-Frequency and Microwave Communication Circuits: Analysis and Design. John Wiley \& Sons, Inc., 2001.

[12]. A. B. Dalby, "Signal transmission on power lines: Analysis of power line circuits," in Proceedings of IEEE Int. Symposium on Power-Line Communications and Its Applications, ser. Essen Germany, 2-4, Apr. 1997, pp. 3744.

[13]. M. N. O. Sadiku, Elements of Electromagnetics, 2nd ed. Oxford University Press, 1995.
[14]. H. Philips, "Performance measurements of power line channels at high frequencies," in Proceedings of Int. Symposium on Power-Line Communications and Its Application ser. Tokyo, Japan, 24-26, Mar. 1998, pp. 229 237.

[15]. A. K. Mandal, Introduction to Control Engineering: Modelling, Analysis and Design. New Age Int., 2006.

[16] N. S. Nise, Control Systems Engineering, 4th ed. John Wiley \& Sons, Inc., 2004.

[17]. Portnov E.L. and et al, "Transmission Lines and Waveguides for Electro-Communication Systems", Garyachi-Liniya Telekom publishers, Moscow, Russian Federation, 2007 (Russian script).

[18]. Hendrik C.F., Luts L., Newbury J., Swart T.G., "Power Line Communications. Theory and Applications for Narrowband \& Broadband over Power Lines", Wiley \& Sons ltd., June 2010.

\section{BIOGRAPHY}

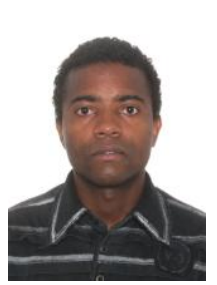

Ndolo A.O. holds MEng (Telecommunication Engineering) with distinction, 2014 and First Class Honours BEngTech (Telecommunication Engineering), 2012 both from Moscow Technical University of Communication and Informatics, Moscow, Russian Federation. He is currently working at Machakos University College (A Constituent of Kenyatta University) as Acting Chairman, Department of Electrical and Electronic Eng. His areas of interest include RF, Optical Communication Systems, RoF, Power Line Communication, and DSP. 